\title{
A CMOS Compatible Ge-on-Si APD Operating in Proportional and Geiger Modes at Infrared Wavelengths
}

\author{
Amir Sammak ${ }^{1}$, Mahdi Aminian ${ }^{2}$, Lin $\mathrm{Qi}^{1}{ }^{1}$, Wiebe D. de Boer ${ }^{1}$, Edoardo Charbon ${ }^{1,2}$ and Lis K. Nanver ${ }^{1}$, \\ ${ }^{1}$ Technische Universiteit Delft, Delft, Netherlands \\ ${ }^{2}$ Ecole Polytechnique Fédérale de Lausanne (EPFL), Lausanne, Switzerland
}

\begin{abstract}
A CMOS compatible Ge avalanche photodiode is fabricated on Si by using a selective chemical-vapor deposition (CVD) epitaxial growth technique. At a temperature of $700^{\circ} \mathrm{C}$, single crystal islands of As-doped Ge are grown in windows to the Si with sizes up to hundreds of $\mu \mathrm{m}^{2}$, to a layer thickness of approximately $1 \mu \mathrm{m}$, This is followed by a pure Ga and then a pure $\mathrm{B}$ (PureGaB) deposition for the creation of an ultrashallow $\mathrm{p}^{+}$-region that can be metallized by Al. The resulting $\mathrm{p}^{+} \mathrm{n}$ diodes have exceptionally good $\mathrm{I}-\mathrm{V}$ characteristics with ideality factors of $\sim 1.1$ and reliably low saturation currents. The measurements show high sensitivity of the photodiodes to IR wavelengths above $1 \mu \mathrm{m}$ in avalanche and Geiger modes.
\end{abstract}

\section{Introduction}

Photon counters that are highly sensitive in the telecom band at $1.55 \mu \mathrm{m}$ are essential in quantum communications for implementing quantum key distribution (QKD) protocols, but also in several imaging techniques based on near infrared sources [1],[2]. State-of-the-art photon counters operating in this band generally require the use of III-IV compounds and alloys, such as InGaAs/InP/InAlAs. The literature on this class of materials is vast; see e.g, [3],[4] as an example. Devices commercially available today based on these technologies can reach quantum efficiencies higher than $50 \%$ and dark count rates of less than $100 \mathrm{~Hz}$ but they are usually cooled to operate at cryogenic temperatures.

Photomultiplier tubes (PMTs) are the oldest technology available but they are still used thanks to their good timing resolution and ease to use, but they are bulky and do not operate in strong magnetic fields, thus limiting their application in some medical imaging fields. Germanium avalanche photodiodes (APDs) have existed for decades but their sensitivity in the telecom band is limited and they exhibit high noise in a wide frequency band, even when operated cryogenically.

All these technologies share a fundamental incompatibility with standard CMOS processes and are thus not indicated for low-cost photon counters and large photon-counting pixel arrays with and without on-chip processing. Silicon photomultipliers (SiPMs) and CMOS single-photon avalanche diodes (SPADs), whose bandgap enables the detection of shorter than $1.1 \mu \mathrm{m}$ wavelengths, cannot be used in the telecom band, unless upconversion techniques are used
[5]. However, such techniques are extremely inefficient and inappropriate in most applications.

In this paper, we propose a new Ge-on-Si APD grown epitaxially on $\mathrm{Si}$ in a manner that is highly compatible with standard Si CMOS processing. The CVD growth technique used to fabricate these $\mathrm{p}^{+} \mathrm{n}$ ultrashallow Ge junction diodes has several exceptionally attractive properties:

- Ge with a very low density of threading dislocations is grown selectively in oxide windows to the $\mathrm{Si}$ that can be tens-of-microns large,

- threading dislocations are trapped within the first $300 \mathrm{~nm}$ of $\mathrm{Ge}$ growth and good quality single-crystal $\mathrm{Ge}$ with a planar surface is achieved for a layer thickness of $1 \mu \mathrm{m}$,

- a nm-thin $\mathrm{p}^{+}$-doping is formed by pure $\mathrm{Ga}$ (PureGa) deposition,

- after Ga deposition a nm-thin pure B (PureB) deposition is performed to create an amorphous B-layer that is a barrier to the Al-metallization, giving reliable contacting and a low series resistance. The term PureGaB is introduced for this combination of pure $\mathrm{Ga} / \mathrm{B}$ depositions,

- no extra perimeter isolation techniques were necessary for obtaining good diode characteristics and avalanching behavior,

- for maximum photon absorption, the Al-metal on the Ge diode surface can be removed selectively to the PureB layer as has been demonstrated for Si photodiodes in [6].

\section{Device fabrication}

The APD fabrication flow is shown in Fig. 1. The starting material is p-type $\mathrm{Si}(100)$ wafers with a resistivity of 2-5 $\Omega$ $\mathrm{cm}$. First a buried $\mathrm{n}^{+}$-layer is implanted and capped with a $0.5-\mu \mathrm{m}$-thick $\mathrm{Si}$ epi-layer. Then $\mathrm{n}^{+}-$and $\mathrm{p}^{+}$-plugs are implanted for contacting the buried n-region and p-substrate, respectively. An isolation layer of $1 \mu \mathrm{m} \mathrm{LPCVD} \mathrm{SiO}_{2}$ is then deposited and those regions where Ge growth is desired are opened by plasma etching to the $\mathrm{Si}$ with soft landing. Selective epitaxial growth of Ge is performed in an ASMI Epsilon $2000 \mathrm{CVD} \mathrm{Si} / \mathrm{SiGe}$ system after a native oxide free Si surface is ensured by doing a HF dip-etch and Marangoni cleaning step followed by a 1 -min bake at $1100^{\circ} \mathrm{C}$ in the reactor itself.

A good quality n-doped $\mathrm{Ge}$ was found to be deposited for a pressure and temperature of 20 Torr and $700^{\circ} \mathrm{C}$, respectively, using a $2 \%$ diluted $\mathrm{GeH}_{4}$ and $0.7 \% \mathrm{AsH}_{3}$ as the precursor gases. These growth conditions were found to render reasonable selectivity to the exposed $\mathrm{Si}$ surface with very little Ge nuclei forming on the oxide. The high quality of 
even large Ge-islands is demonstrated by the TEM/SEM images of Fig. 2 and Fig. 3.

In order to create a $\mathrm{p}^{+} \mathrm{n}$ junction, a novel processing procedure was developed for the fabrication of the $\mathrm{p}^{+} \mathrm{Ge}$ by a sequence of in-situ pure $\mathrm{Ga}$ and then pure $\mathrm{B}$ (PureGaB) depositions using TMGa and $\mathrm{B}_{2} \mathrm{H}_{6}$ as precursors. While pure $\mathrm{Ga}$ produces an effective ultrashallow $\mathrm{p}^{+}$doping on the surface, the pure B layer protects the surface against further oxidation as well as Al-spiking [7],[8].

For the metallization, physical-vapor-deposited (PVD) $\mathrm{Al} / \mathrm{Si}(1 \%)$ is used to contact the n-type $\mathrm{Si}$ and the anode of the $\mathrm{p}^{+} \mathrm{n}$ Ge diode. Arrays of diodes with different areas, as large as $40 \times 40 \mu \mathrm{m}^{2}$, were fabricated. They all displayed good I-V characteristics and the smaller ones could be operated in Geiger mode. In the following, two APDs with areas $2 \times 2$ and $2 \times 20 \mu \mathrm{m}^{2}$ are characterized.
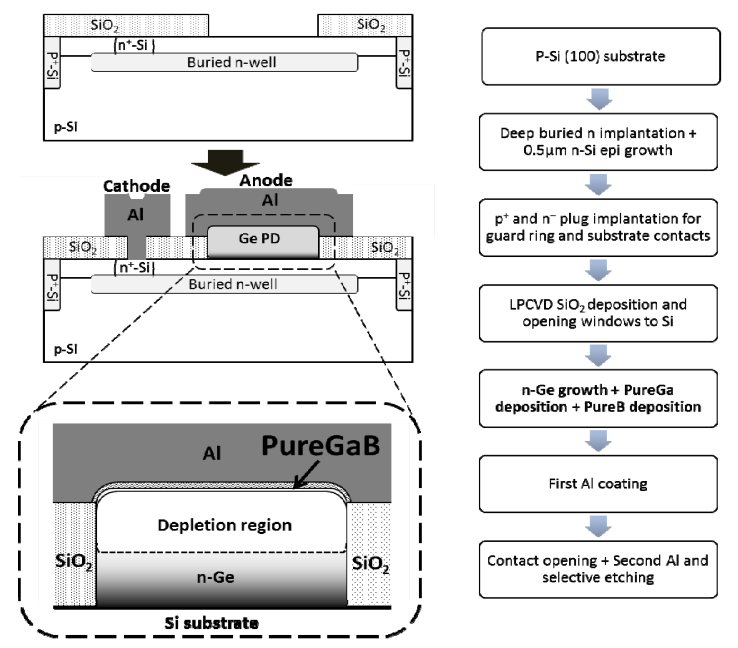

Fig. 1. APD fabrication flowchart.

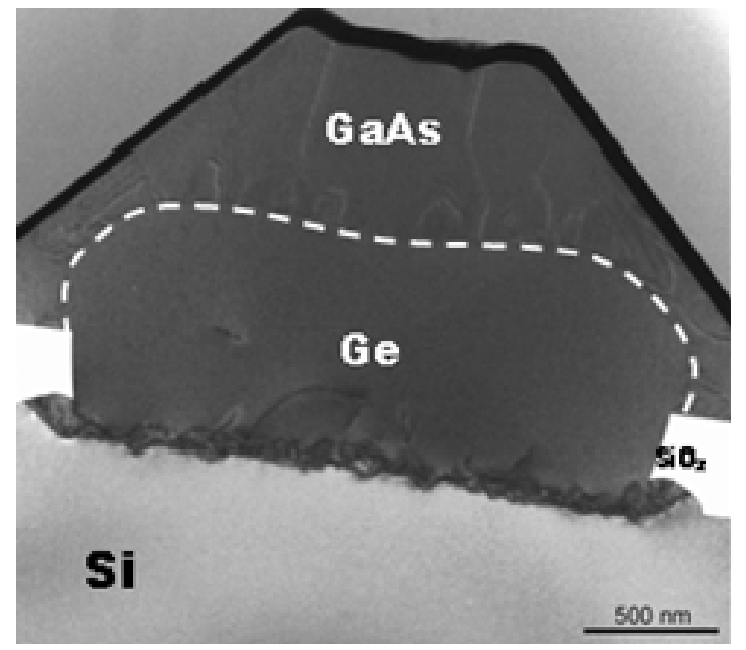

Fig. 2. Cross-sectional TEM image of a Ge-island epitaxially grown at $700^{\circ} \mathrm{C}$ on patterned Si followed by in-situ growth of a GaAs layer. Due to the rounded form of the Ge surface, the GaAs growth is not entirely free of dislocations [7].

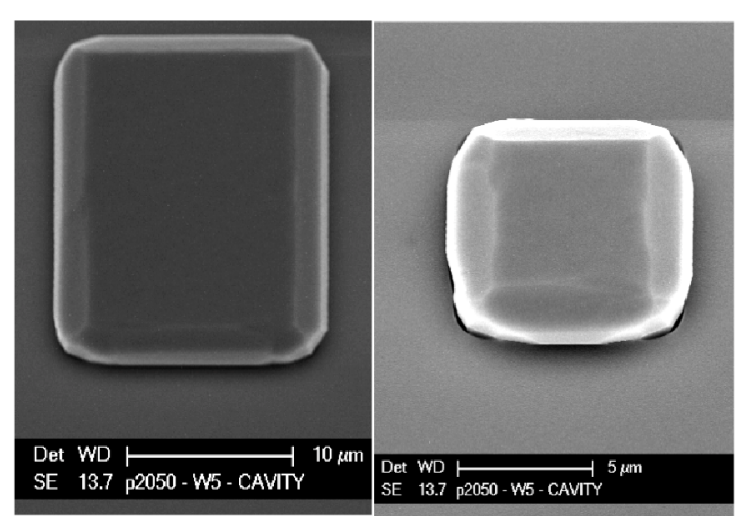

Fig. 3. Top-view SEM images of Ge-islands grown on a $\mathrm{Si}$ substrate as described in the APD flowchart given in Fig. 1, taken before metallization.

\section{Optical/Electrical Characterization}

The current-voltage (I-V) characteristics of photodiodes with two different sizes are shown in Fig. 4. The breakdown voltages, $\mathrm{V}_{\mathrm{BD}}$, are found to be

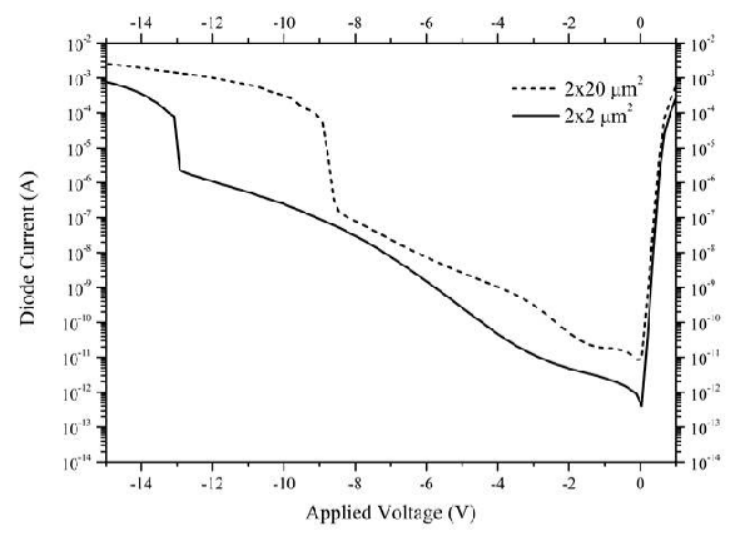

(a)

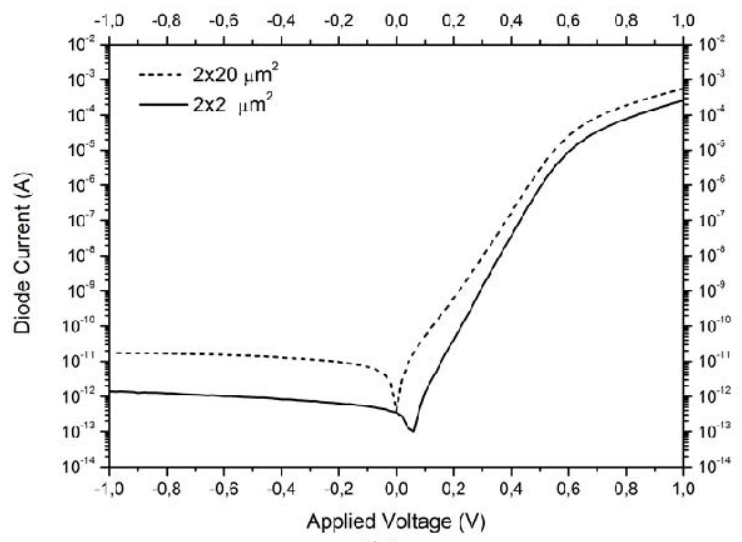

(b)

Fig. 4. I-V characteristics of APDs with areas of $2 \times 2 \mu \mathrm{m}^{2}$ and $2 \times 20 \mu \mathrm{m}^{2}$. The breakdown is shown in (a) and the ideality factors are, respectively, 1.15 and 1.35 . 
respectively $13 \mathrm{~V}$ and $9 \mathrm{~V}$ for the $2 \times 2 \mu \mathrm{m}^{2}$ and $2 \times 20 \mu \mathrm{m}^{2}$ devices and the corresponding ideality factors are 1.15 and 1.35. The dark currents at $1 \mathrm{~V}$ reverse bias are as low as $2 \mathrm{pA}$ and $20 \mathrm{pA}$ for the $2 \times 2 \mu \mathrm{m}^{2}$ and $2 \times 2 \mu \mathrm{m}^{2}$ devices, respectively, and increase to hundreds of micro-amps past the breakdown. In Fig. 5 a schematic is shown of the circuit used to operate the APD in Geiger mode with passive quenching and passive recharge via a ballast resistor. The APD is biased above breakdown $\left(\mathrm{V}_{\mathrm{BD}}\right)$ by a voltage known as the excess bias $\left(\mathrm{V}_{\mathrm{e}}\right)$, The total voltage is $\mathrm{V}_{\mathrm{OP}}=\left|\mathrm{V}_{\mathrm{BD}}\right|+\mathrm{V}_{\mathrm{e}}$. The range of $\mathrm{V}_{\mathrm{e}}$ is from 0 to $4 \mathrm{~V}$. This high operational voltage causes high electric fields across the depleted $\mathrm{p}^{+}-\mathrm{n}$ junction that may cause an avalanche current when the interaction of a photon or a thermal event in the Ge creates electron-hole pairs.

The $\mathrm{p}^{+}$-anode of the APD is connected to the ballast resistor. Whenever a photon reaches the APD, a Geiger pulse is generated, and the ballast resistor quenches the device to be ready for the next event. The avalanche current going through this resistor builds up to a sharp voltage pulse, known as a Geiger event. Geiger events are converted to digital pulses by using a comparator with an appropriate threshold voltage $\mathrm{V}_{\text {th }}$.

Besides photon-generated pulses, in the dark, carriers can be generated due to generation-recombination processes inside and near the multiplication region. This results in dark counts, characterized by the parameter dark count rate (DCR), or the mean frequency of dark counts; it is measured as a function of excess bias voltage and is plotted in Fig. 6 for the two devices.

In Fig. 7 the photo-response is given for the $2 \times 20 \mu \mathrm{m}^{2}$ detector in sub-Geiger mode (both in linear mode and in proportional APD mode). The breakdown voltage is near $11 \mathrm{~V}$ and the current after breakdown $(\sim 100 \mu \mathrm{A})$ is 500 times bigger than proportional APD mode current of 200nA. The IR-induced current is higher than that induced by visible light which confirms that these Ge photodiodes are more sensitive in the IR region.

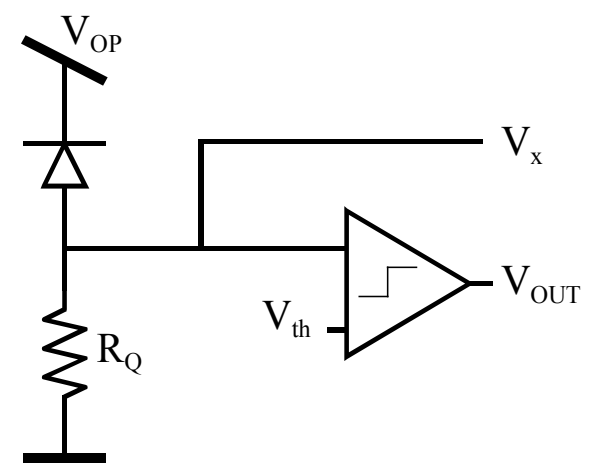

Fig. 5. The APDs were biased above breakdown using the above circuit, so as to operate in Geiger mode. The ballast resistance $R_{Q}$ is used to perform passive quenching and passive recharge. $V_{O P}=\left|V_{B D}\right|+V_{e}$, where $V_{e}$ is the excess bias voltage and $\mathrm{V}_{\mathrm{BD}}$ is the breakdown voltage. The comparator, with an appropriate threshold voltage $\mathrm{V}_{\text {th }}$, was used to convert Geiger pulses to digital signals.

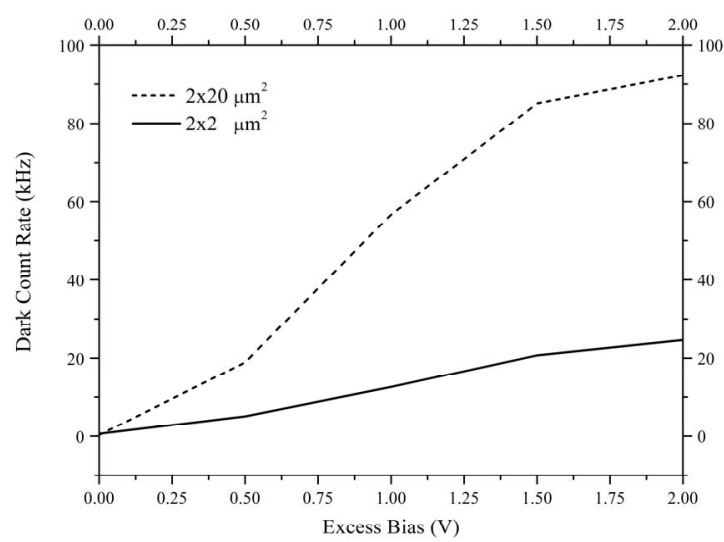

Fig. 6. Dark count rate (DCR) as a function of excess bias voltage $V_{e}$, at room temperature.

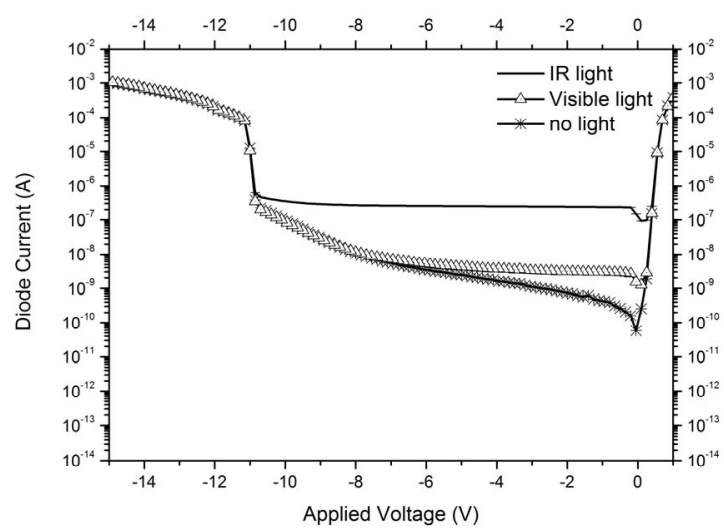

Fig. 7. Photo response of the $2 \times 20 \mu \mathrm{m}^{2}$ APD as a function of reverse bias when exposed to a wideband $800-\mathrm{nm}$ source.

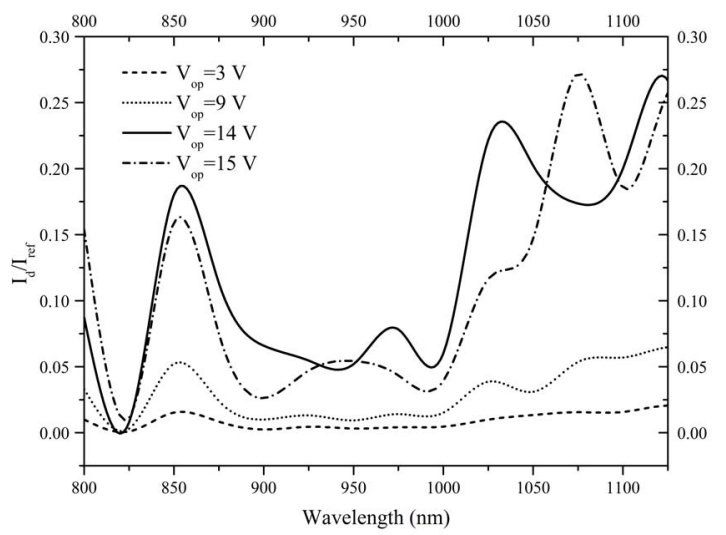

Fig. 8. $I_{d} / I_{\text {ref }}$ of the $2 \times 20 \mu m^{2}$ APD in the NIR spectrum for various bias voltages $\mathrm{V}_{\mathrm{OP}}$.

To evaluate the sensitivity of photon detection in avalanche and Geiger modes, the photocurrent $I_{d}$ of the $2 \times 20 \mu \mathrm{m}^{2}$ diode is measured at different wavelengths by illumination with a light-source spot that is much larger than the diode area. The $I_{d}$ is compared with the photocurrent $I_{\text {ref }}$ measured on a 
reference photodiode with an area larger than the spot size for which the quantum efficiency $\mathrm{QE}$ is known for all wavelengths of interest. The ratio $I_{d} / I_{\text {ref }}$ is plotted as a function of wavelength in Fig. 8 for the bias voltages of $3 \mathrm{~V}$ and 9V (below breakdown) and 14V and 15V (Geiger mode). The $25 \%$ peak at an IR-wavelength of $1100 \mathrm{~nm}$ in Geiger mode is measured for excess bias voltages of $3 \mathrm{~V}$ and $4 \mathrm{~V}$. This gives a conservative indication of the high avalanche current obtainable with the PureGaB diodes.

The timing response is plotted in Fig. 9 for the APD when exposed to a pulsed laser that was centered at $637 \mathrm{~nm}$ (Advanced Laser Diode Systems GmBH, Berlin, Germany) with an excess bias voltage of $1 \mathrm{~V}$. The measurement was obtained using the embedded time discriminator of a LeCroy WaveMaster 8600A. This jitter performance was measured for the $2 \times 2 \mu \mathrm{m}^{2}$ APD with a pulse width of $80 \mathrm{ps}$. The measured full width at half maximum (FWHM) jitter was 900ps.

The performance characterization of the photodiodes in the three modes of operation (linear, APD, and Geiger) is summarized in Tab. 1. The devices can be fabricated with a range of breakdown voltages that can be accurately controlled from a minimum of $9 \mathrm{~V}$ to a maximum of $13 \mathrm{~V}$. The excess biases of 0 to $4 \mathrm{~V}$ were applied to the APDs for DCR measurements.

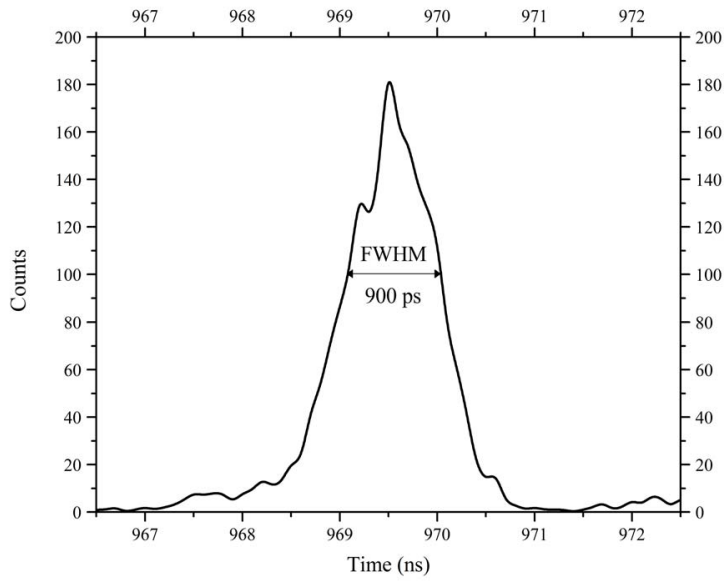

Fig. 9. Jitter performance of the $2 \times 2 \mu \mathrm{m}^{2}$ APD when operated in Geiger mode. The APD was illuminated by a pulsed laser source at a wavelength of $637 \mathrm{~nm}$ with a pulse width of $80 \mathrm{ps}$. The measured FWHM jitter was less than 1000 ps.

\section{Conclusions}

The fabricated Ge APD detectors can be operated both in proportional and in Geiger mode. They exhibit relatively low dark counts and high sensitivity at room temperature. This correlates well with the fact that the I-V characteristics have, to our knowledge, uniquely low values of reverse current, series resistance and ideality factors as well as good uniformity over the wafer.
Tab. 1. Summary of performance measurements for linear mode, APD mode, and Geiger mode of operation

\begin{tabular}{l|l|l|l|l}
\hline Performance (APD/linear mode) & Min. & Typ. & Max. & Unit \\
\hline Dark current @ 1V reverse bias & 2 & & 20 & $\mathrm{pA}$ \\
\hline Active area & 4 & & 40 & $\mu \mathrm{m}^{2}$ \\
\hline Breakdown voltage & 9 & 11 & 13 & $\mathrm{~V}$ \\
\hline
\end{tabular}

\begin{tabular}{l|l|l|l|l}
\hline Performance (Geiger mode) & & & & \\
\hline $\mathrm{DCR} @ \mathrm{Ve}=1 \mathrm{~V}$ & 10 & & 60 & $\mathrm{kHz}$ \\
\hline $\mathrm{I}_{\mathrm{d}} / \mathrm{I}_{\mathrm{ref}} @ \mathrm{~V}_{\mathrm{e}}=3 \mathrm{~V}$ & & & 25 & $\%$ \\
\hline FWHM Time jitter & & & 900 & $\mathrm{ps}$ \\
\hline Excess bias voltage & 0 & & 4 & $\mathrm{~V}$ \\
\hline
\end{tabular}

\section{Acknowledgements}

The authors would like to thank the staff of the DIMES cleanrooms for processing support as well as the staff of the DIMES measurement room and the Photovoltaic Materials/Devices group for respectively electrical and optical measurement support. This project has received financial support from the SmartMix Memphis project and the NanoTera-IrSens project.

\section{References}

[1] J.H. Shapiro, N.C. Wong, J. Opt. B: Quantum Semiclass. Opt., 2, L1-L4 (2000).

[2] D. Stucki, G. Ribordy, A. Stefanov, H. Zbinden, J. Rarity, T. Wall, J. Mod. Opt., 48, 1967 (2001).

[3] M.A. Itzler, X. Jiang, R. Ben-Michael, B. Nyman, K. Slomkowski, IEEE Sel. Top. Quantum Electron., 13(4), 849-1038 (2007).

[4] P. Yuan, J. Boisvert, R. Sudharsanan, T. Isshiki, P. McDonanld, M. Salisbury, M. Liu, J. Campbell, Proc. of SPIE 6900, 69001B (2008).

[5] R. T. Thew, S. Tanzilli, L. Krainer, S. C. Zeller, A. Rochas, I. Rech, S. Cova, H. Zbinden, and N. Gisin, New J. Phys., 8, 32 (2006).

[6] A. Sakic, L.K. Nanver, G. Van Veen, K. Kooijman, P. Vogelsang, T.L.M. Scholtes, W. De Boer, W.H.A. Wien, S. Milosavljevic, C.Th.H Heerkens, T. Kneževi and I. Spee, IEDM2010 39(2), 162-173, 2010.

[7] A. Sammak, W. D. deBoer, L. Qi and L. K. Nanver, in Proceedings of IEEE 41st European Solid-State Device Research Conference (ESSDERC 2011), in press.

[8] A. Sammak, L. Qi, W. D. deBoer and L. K. Nanver, 10th IEEE International Conference on Solid-State and Integrated Circuit Technology (ICSICT 2010), Shanghai, China, pp. 969-971 